\title{
EHMTI-0275. Headache and cervical spine disturbances in a patient with osteoarthritis of temporomandibular joint - one-year-follow-up
}

\author{
T Badel $^{1 *}$, V Basic Kes ${ }^{2}$, D Rosic ${ }^{3}$, D Zadravec ${ }^{4}$, J Kern $^{5}$ \\ From 4th European Headache and Migraine Trust International Congress: EHMTIC 2014 \\ Copenhagen, Denmark. 18-21 September 2014
}

\section{Introduction}

Temporomandibular joint (TMJ)-related pain also includes the craniomandibular and cervicocranial areas due to the topographic-functional closeness.

\section{Aims}

To determine the relationship between types of headaches and cervical spine (CS) disorders in a patients with osteoarthritis (OA) of TMJ with one-year-follow-up.

\section{Methods}

65 patients (mean age 47.4, 95.4\% women) were consecutively treated for signs and symptoms of OA of TMJ. A definitive diagnosis of OA was confirmed by magnetic resonance imaging. The patients were examined by a dentist, a neurologist, and a physiatrist-rheumatologist. Pain intensity in TMJ was shown on the visual-analogue scale (VAS 0-10). They were treated by an occlusal splint and physical therapy with one-year-follow-up.

\section{Results}

The applied treatment modalities achieved a significant reduction of pain $(\mathrm{p}<0.001)$ in the TMJ at first examination and one-year-follow-up (mean values on VAS: 6.58 and 1.67). 46.2\% of patients did not have a CS diagnosis and $53.9 \%$ of patients did not have headaches. $16.9 \%$ of them had migraines, $23.1 \%$ had CS-related headache and $6.1 \%$ of patients had tension-types headaches. Cervical syndrome was found in $10.8 \%$ of patients. $26.1 \%$ had cervicobrachial syndrome, $7.7 \%$ had cervicocephalic syndrome and $9.2 \%$ of patients had both. The SC syndrome was significant regarding the patients' age (mean age 40.8 as opposed to 53.1 years, with $\mathrm{p}=0.0002$ ), whereas there were no differences for headaches.

\section{Conclusions}

The relationship of SC disturbances with the higher age of patients was determined in patients with OA of TMJ. The existence of comorbidity with headaches does not affect treatment success of TMJ.

No conflict of interest.

\section{Authors' details}

'Department of Removable Prosthodontics, School of Dental Medicine,

Zagreb, Croatia. ${ }^{2}$ Department of Neurology, Clinical Hospital Center Sestre milosrdnice, Zagreb, Croatia. ${ }^{3}$ Department for Rheumatic Disease, Center for Rheumatic Disease Physical Therapy and Rehabilitation "dr Drago Cop", Zagreb, Croatia. "Department of Diagnostic and Interventional Radiology, linical Hospital Center Sestre milosrdnice and Rehabilitation "dr Drago Cop", Zagreb, Croatia. ${ }^{5}$ Department of Medical Statistics Epidemiology and Medical Informatics, School of Public Health "Andrija Štampar", Zagreb, Croatia.

Published: 18 September 2014

doi:10.1186/1129-2377-15-S1-C5

Cite this article as: Badel et al:: EHMTI-0275. Headache and cervical spine disturbances in a patient with osteoarthritis of temporomandibular joint - one-year-follow-up. The Journal of Headache and Pain 2014 15(Suppl 1):C5. 